Astrophysics and Space Science manuscript No.

(will be inserted by the editor)

Daniel Mazin for the MAGIC collaboration

\title{
Observations of Extragalactic Sources with the MAGIC Telescope
}

TeV Blazars and Extragalactic Background Light

Received: date / Accepted: date

\begin{abstract}
MAGIC is currently the world's largest single dish ground based imaging atmospheric Cherenkov telescope. During the first year of operation, more than 20 extragalactic sources have been observed and several of them detected. Here we present results of analyzed data, including discussion about spectral and temporal properties of the detected sources. In addition, we discuss implications of the measured energy spectra of distant sources for our knowledge of the extragalactic background light.
\end{abstract}

Keywords TeV gamma-ray astrophysics · blazars · EBL

PACS 95.85.Pw $\cdot 98.54 . \mathrm{Cm} \cdot 98.70 . \mathrm{Vc}$

\section{Introduction}

The search for very high energy (VHE, defined as $E \geq 100$ $\mathrm{GeV}) \gamma$-ray emission from Active Galactic Nuclei (AGNs) is one of the major goals for ground-based $\gamma$-ray astronomy. New detections open up a possibility of phenomenological studies of the physics inside the relativistic jets in AGNs, in particular of understanding both the origin of the VHE $\gamma$-rays as well as the relations between photons of different energy (from radio to VHE). The number of extragalactic $\gamma$ ray sources detected by EGRET on board the CGRO with high confidence amounts to 66 [1]. However, the number of AGNs reported to be VHE $\gamma$-ray emitters (although slowly increasing) is currently just thirteen (July 2006).

Stecker et al. [2] pointed out that the attenuation of $\gamma$ rays due to photon-photon interactions with low energy photons from the extragalactic background light (EBL) was a likely explanation of this deficit. In fact, the redshifts of blazars detected so far above $100 \mathrm{GeV}$ have rather low values as expected from predictions of the correlation between

Daniel Mazin

Max-Planck-Institut für Physik

Föhringer Ring 6

D-80805 München

Tel.: +49-89-32354259

Fax: +49-89-32354516

E-mail: mazin@mppmu.mpg.de the gamma-ray attenuation and the redshift of the source, known as Fazio-Stecker relation [3 4].

All known VHE gamma-ray emitting blazars belong to the class of high-frequency-peaked BL Lacertae objects (HBLs, [5]), a subclass of blazars characterized by a low luminosity when compared with quasars and a synchrotron peak in the X-ray band. Their Spectral Energy Distribution (SED) is characterized by a second peak at very high gammaray energies. In synchrotron-self-Compton (SSC) models it is assumed that the observed gamma-ray peak is due to the inverse-Compton (IC) emission from the accelerated electrons up-scattering previously produced synchrotron photons to high energies [6]. A compilation of blazars with known Xray spectra allowing their classification as HBLs is given in [7].

The Major Atmospheric Gamma-ray Imaging Cherenkov (MAGIC) telescope observed a sample of X-ray bright $\left(F_{1 \mathrm{keV}}>2 \mu \mathrm{Jy}\right)$ northern HBLs at moderate redshifts $(z<0.3)$. The sample of candidates was chosen based on predictions from models involving an SSC [8] and hadronic [9] origin of the $\gamma$-rays.

The known VHE $\gamma$-ray emitting AGNs are variable in flux in all wavebands. Correlations between X-ray and $\gamma$-ray emission have been found on time scales ranging from several 10 minutes to days (e.g. [10]) although the relationship has proven to be rather complicated. The optical-TeV correlation has yet to be studied, but the optical-GeV correlations seen in 3C 279 [12] suggest that at least in some sources such correlations do exist. Using this as a guideline, the MAGIC collaboration has performed Target of Opportunity observations whenever they were alerted about sources being in a high flux state in the optical and/or X-ray band.

Here we present results of the detected extragalactic sources ordered by increasing redshift values: Mkn 421 ( $\mathrm{z}=0.030), \mathrm{Mkn} 501 \quad(\mathrm{z}=0.034), 1 \mathrm{ES} 2344+514 \quad(\mathrm{z}=0.044)$, Mkn $180(\mathrm{z}=0.045), 1 \mathrm{ES} 1959+650(\mathrm{z}=0.047), 1 \mathrm{ES} 1218+304$ ( $\mathrm{z}=0.182$ ), PG 1553+113 (unknown redshift, $\mathrm{z}>0.09$ [13]). The results from galactic observations are presented elsewhere in these proceedings [14]. 

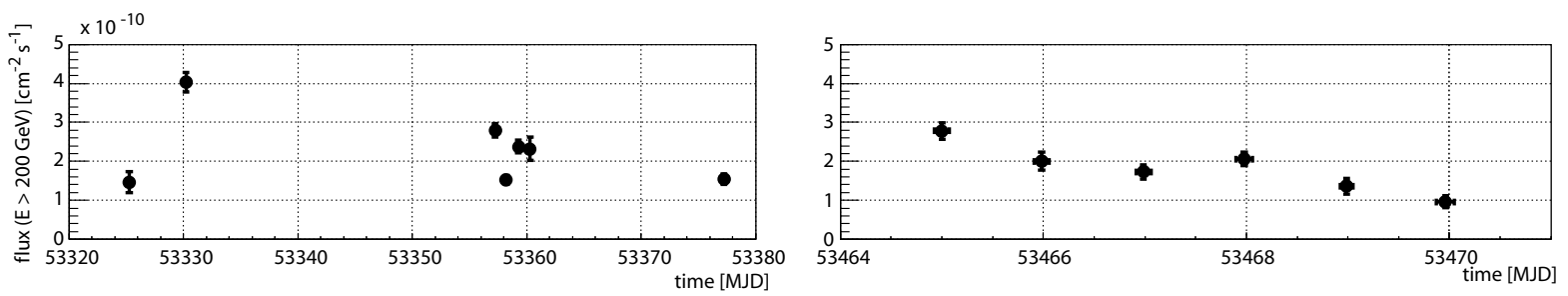

Fig. 1 Lightcurve for Mkn 421 from November 2004 to April 2005. Left panel: data from November 2004 to January 2005. Right panel: data for April 2005.

\section{MAGIC}

The MAGIC telescope [15] is located on the Canary Island La Palma ( $2200 \mathrm{~m}$ asl, $\left.28^{\circ} 45^{\prime} \mathrm{N}, 17^{\circ} 54^{\prime} \mathrm{W}\right)$ and is currently the largest imaging atmospheric Cherenkov telescope worldwide. The accessible energy range spans from $50-60 \mathrm{GeV}$ (trigger threshold at small zenith angles) up to tens of $\mathrm{TeV}$. The sensitivity of MAGIC is $\sim 2.5 \%$ of the Crab Nebula flux in 50 hours of observations. The energy resolution is about $30 \%$ above $100 \mathrm{GeV}$ and about $25 \%$ above $200 \mathrm{GeV}$. The $\gamma$ point spread function is about 0.1 degrees.

The standard operation mode for MAGIC is the ONobservation, with the source position in the center of the camera. Usually, to get a robust estimate of the background, part of the data are taken in the OFF mode, where a sky region is tracked by the telescope which resembles the region around the source with respect to the level of the night sky background fluctuations. Part of the data were taken in the so-called WOBBLE mode [16]. In this mode, two sky directions, opposite and $0.4^{\circ}$ off source respectively, were tracked alternately for 20 minutes each. The advantage of the WOBBLE tracking mode is a simultaneous measurement of the background, and thus a priori no need for additional OFF data.

For calibration, image cleaning, cut optimization, and energy reconstruction, the MAGIC standard analysis chain [17] was used. For $\gamma /$ hadron separation as well as for energy determination a multidimensional classification technique based on the Random Forest method [18 19] was used. The cuts were then chosen such that the overall cut efficiency for Monte-Carlo $\gamma$ events was about $50 \%$. The systematic errors were estimated to be around $50 \%$ for the absolute flux level and 0.2 for the spectral index.

In parallel with the observations of the AGNs with MAGIC, the sources were observed with the KVA $35 \mathrm{~cm}$ telescope (http://tur3.tur.iac.es/), also located on La Palma. In addition to these joint campaigns, several AGNs are regularly observed as part of the Tuorla Observatory Blazar monitoring program with the Tuorla $1 \mathrm{~m}$ and the KVA $35 \mathrm{~cm}$ telescopes.

In the following sections we present the highlights of the extragalactic observations by the MAGIC telescope. The observational details of the selected sources including an analysis energy threshold are summarized in Table 1
Table 1 Summary of observation details of the presented data. ZA is the zenith angle in degrees. $\mathrm{E}_{\mathrm{Thr}}$ is the analysis threshold in $\mathrm{GeV}$. T(hr) is the observation time in hours.

\begin{tabular}{lllll}
\hline Source & Period & ZA & $\mathbf{E}_{\text {Thr }}$ & T(hr)/Mode \\
\hline \multirow{2}{*}{ Mkn 421 } & Nov04-Apr05 & $9-55$ & \multirow{2}{*}{150} & 15.5/ON \\
& Apr05 & $9-32$ & & 10.1/WOBBLE \\
\hline Mkn 501 & Jun-Jul05 & $10-31$ & 150 & $29.7 / \mathrm{ON}$ \\
\hline 1ES 2344 & Sep-Dec05 & $23-34$ & 160 & $27.6 / \mathrm{WOBBLE}$ \\
\hline Mkn 180 & Mar06 & $39-44$ & 200 & $11.1 / \mathrm{WOBBLE}$ \\
\hline 1ES 1959 & Sep-Oct04 & $36-46$ & 180 & $6.0 / \mathrm{ON}$ \\
\hline 1ES 1218 & Jan05 & $2-13$ & 140 & $8.2 / \mathrm{ON}$ \\
\hline PG 1553 & Apr-May05 & $12-30$ & 140 & $7.0 / \mathrm{ON}$ \\
& Jan-Apr06 & $20-30$ & & $11.8 / \mathrm{ON}$ \\
\hline
\end{tabular}

\section{Markarian 421}

Mkn 421 (redshift $z=0.030$ ) is the closest known TeV blazar and, along with Mkn 501, also the best studied one. It was the first extragalactic $\gamma$-ray source detected in the TeV energy range using IACTs [20]. So far, Mkn 421 has shown variations larger than one order of magnitude and occasional flux doubling times as short as $15 \mathrm{~min}$ [21]. Variations in the hardness of the $\mathrm{TeV} \gamma$-ray spectrum during flares were reported by several groups (e.g. [22,23]). Simultaneous observations in the X-ray and GeV-TeV bands showed a significant flux correlation [24].

Mkn 421 was observed with the MAGIC for a total of 19 nights, the observation times per night ranging from 30 minutes up to 4 hours (Table 1 ). Most of the data were taken at small zenith angles $\left(Z A<30^{\circ}\right)$. Only 1.5 hours in December 2005 were taken at $42^{\circ}<Z A<55^{\circ}$ during simultaneous observations with H.E.S.S. [25].

During the entire observation period Mkn 421 was found to be in a medium flux state ranging from 0.5 to $2 \mathrm{Crab}$ units above $200 \mathrm{GeV}$ (see Fig. 11). Significant variations of up to a factor of four overall and up to a factor two in between successive nights can be seen. Fig. 2 shows a clear correlation between X-ray (taken from the All-Sky-Monitor on-board the RXTE satellite) and VHE $\gamma$-ray data. The points are the nightly average of the MAGIC data and the simultaneous ASM count rate. A linear fit as well as a parabolic fit are forced to go through $(0,0)$ and describe the correlation well. The linear correlation coefficient is $r=0.64_{-0.22}^{+0.15}$.

The measured spectrum and the reconstructed deabsorbed (i.e. corrected for the effect of extragalactic ab- 


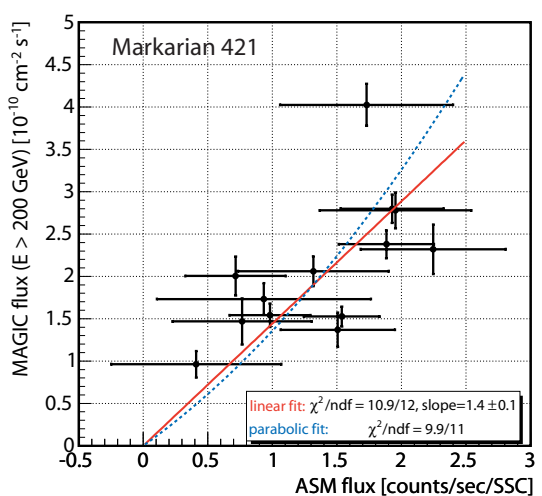

Fig. 2 Correlation plot between VHE $\gamma$-ray flux above $200 \mathrm{GeV}$ and $\mathrm{X}$-ray counts for 11 nights of Mkn 421 data.

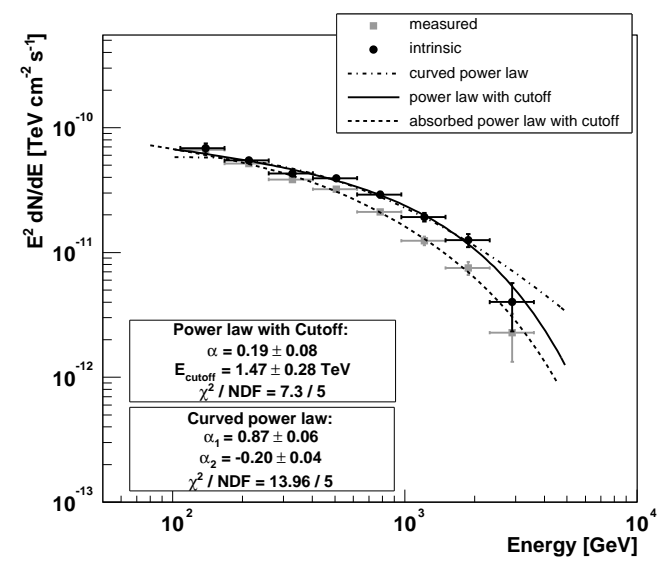

Fig. 3 The measured (grey points) and de-absorbed (black points) spectrum of Mkn 421, multiplied by $E^{2}$. Solid line: fit (1) to the intrinsic spectrum using a power law with a cut-off. Dashed-dotted line: fit (2) to the intrinsic spectrum using a curved power law. A dashed line indicates the expected absorbed spectrum using the result of fit (1). Fit parameters of the intrinsic spectrum are shown in the inlays.

sorption) spectrum are shown in Fig. 3 Here we used the recent model of Primack et al. [26], which is in agreement with the upper limits set by H.E.S.S. [45] and low limits from the galaxy counts [27 28]. The de-absorbed spectrum is clearly curved. Therefore, it is obvious that the curvature in the measured spectrum has an intrinsic origin rather than being caused by the absorption of the VHE $\gamma$-rays by the EBL photons. A fit with a pure power law with a exponential cut-off as well as a fit with a curved power law indicate a flattening of the spectrum towards $100 \mathrm{GeV}$. For details of the analysis and results see [29].

\section{Markarian 501}

The AGN Mkn 501 [30] is one of the best-studied objects in VHE $\gamma$-rays. The source is known to be a strong and variable VHE $\gamma$-ray emitter. During a flare in 1997, Mkn 501 showed strong variability on timescales of 0.5 days. The integral flux reached 10 times the flux of the Crab nebula above $1 \mathrm{TeV}$

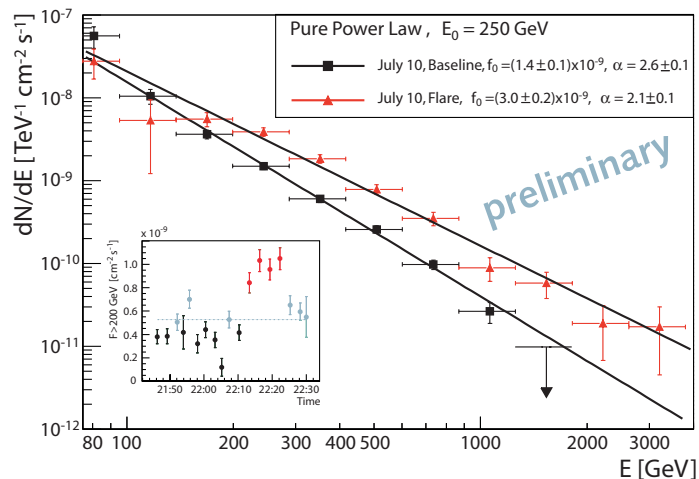

Fig. 5 Spectral differential energy distribution of Mkn 501 on 10 July 2005. The rapid variation of the flux level and corresponding change in the shape of the energy spectrum on a time scale of some 10 minutes is clearly visible.

[31]. The position of the IC peak was not detected yet while it was observed that the spectrum gets harder with an increasing flux level.

Mkn 501 was observed for 24 nights (Table 1) by the MAGIC telescope. It is worth noticing that $\sim 18$ out of 30 hrs of the observation time were performed in the presence of (moderate) moonshine. The source was found to be in a rather low flux state during most of the observations. The flux level above $200 \mathrm{GeV}$ was $30 \%-50 \%$ of the Crab Nebula flux with a strong indication of an IC peak. On five nights, the source was found in a flaring state with the flux reaching up to 4 Crab units (Fig 4). Moreover, a rapid flare with a doubling time as short as 5 minutes or less was detected on the night of 10 July 2005 (inlay in Fig. 5). No change in background rates was seen during the observed flare. The rapid increase in the flux level was accompanied by a hardening of the differential spectrum (Fig. 5). This is the first time that spectral hardening was detected on time scales of some 10 minutes. A detailed publication on the analysis and results on the observation of Mkn 501 is in preparation.

\section{1ES 2344+514}

1ES 2344+514 was identified as a BL Lac object by [32], who also determined a redshift of $z=0.044$ from absorption line measurements, while no evident emission lines were found. In VHE $\gamma$-rays, the source was observed by the Whipple collaboration during the 1995/96 observing season, yielding a signal on the $5.8 \sigma$ level [33]. Over $1 / 3$ of the measured excess was recorded during the night of December 20, 1995, with corresponding photon flux above $350 \mathrm{GeV}$ of $64 \%$ of the Crab nebula flux. The VHE $\gamma$-ray emission of $1 \mathrm{ES} 2344+514$ was confirmed on a significance level of $4.4 \sigma$ by the HEGRA collaboration [34], who found a quiescent flux level approximately 50 times lower than during the 1995 flare.

The MAGIC observation of 1ES 2344+514 (Table 1) yielded a clear excess with the significance of $11.5 \sigma$. The 


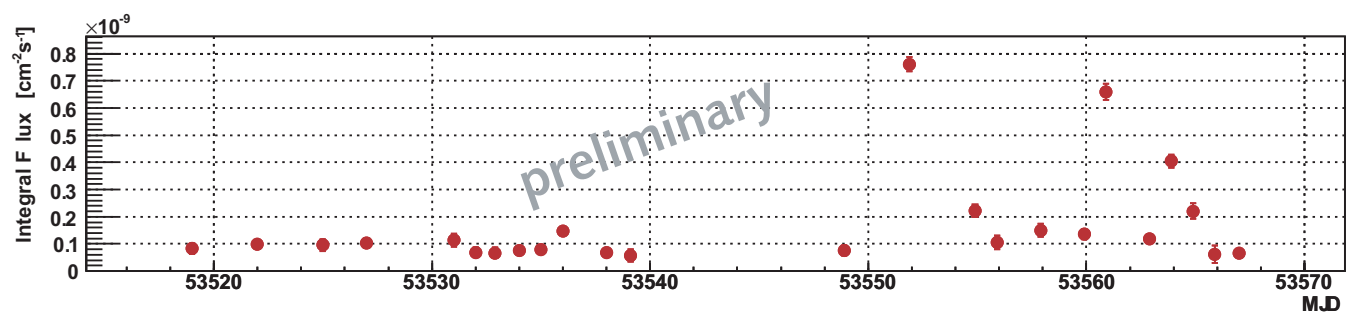

Fig. 4 Lightcurve for Mkn 501 from June-July 2005. Shown are night averaged integral fluxes above $200 \mathrm{GeV}$.

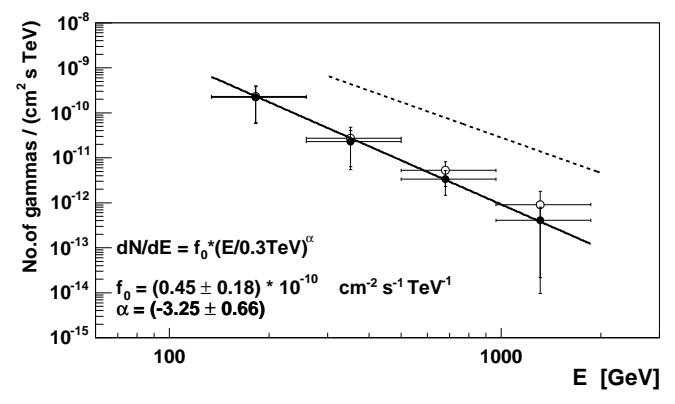

Fig. 6 The differential energy spectrum of Mkn 180. Full circles: the spectrum measured by MAGIC. Open circles: the de-absorbed energy spectrum (see text). The horizontal bars indicate the size of each energy bin. The black line represents a power law fit to the measured spectrum. The fit parameters are listed in the figure. For comparison, the Crab Nebula energy spectrum as derived from MAGIC data [17] is shown (dashed line).

measured flux corresponds to $6 \%$ of the Crab Nebula flux. No strong evidence for flux variability on times scales of days or weeks was found. The differential energy spectrum can be fitted by a simple power law with a photon index of $2.96 \pm 0.12$.

This was the first time that VHE $\gamma$-rays from 1ES 2344+ 514 were detected with high significance in a quiescent flux state. The derived spectrum is softer than the one reported by the Whipple collaboration during the flare in 1995 [35. A detailed publication on the analysis and results of MAGIC observations of $1 \mathrm{ES} 2344+514$ is in preparation.

\section{Markarian 180}

The AGN Mkn 180 (1ES 1133+704) is a well-known HBL at a redshift of $z=0.045$ [36]. Previous attempts to detect VHE $\gamma$-rays only resulted in upper limits [34 37].

The observation of Mkn 180 was triggered by a brightening of the source in the optical on March 23, 2006, detected by the KVA telescope. The alert was given as the core flux increased by $50 \%$ from its quiescent level value. Mkn 180 was observed by the MAGIC telescope in 2006 during 8 nights (Table 1). The signal of 165 excess events was found with a significance of $5.5 \sigma$. No evidence for flux variability between nights was found. The measured energy spectrum of Mkn 180 is shown in Fig. 6 A fit by a power law gives a photon index $\alpha=3.3 \pm 0.7$. The observed integral flux above $200 \mathrm{GeV}$ is $11 \%$ of the Crab Nebula flux. The attenuation of the spectrum caused by the EBL was determined by numerical integration of Eq. 2 in [38]. The de-absorbed energy spectrum of Mkn 180 is also shown in Fig. 6) (open circles). A fit with a simple power law to the de-absorbed spectrum gives a slope with $\alpha^{\prime}=2.8 \pm 0.7$.

The discovery of VHE emission from Mkn 180 during an optical outburst makes it very tempting to speculate about the connection between optical activity and increased VHE emission. Since Mkn 180 has not been observed with MAGIC prior to the outburst and the upper limits from other experiments are above the observed flux level, further observations are needed. For details of this analysis and results see [39].

\section{1ES 1959+650}

The Seven Telescope Array in Utah reported for the first time a VHE $\gamma$-ray signal from 1ES 1959+650 in 1998 with the significance of $3.9 \sigma$ [40]. In May 2002, when the Xray flux of the source had significantly increased, both the Whipple [11] and HEGRA [41] collaborations subsequently confirmed 1ES 1959+650 as a VHE $\gamma$-ray source. An interesting aspect of the source activity in 2002 was the observation of a so-called orphan flare, i.e. a VHE $\gamma$-ray activity is observed in the absence of high activity in X-rays. Orphan flare in VHE $\gamma$-rays are not expected within the SSC mechanism in relativistic jets.

1ES 1959+650 was observed by MAGIC during the comissioning phase in 2004 (Table 1). The analysis of the data gave a detection of VHE $\gamma$-rays on a significance of $8.2 \sigma$. We obtained an integral VHE $\gamma$ flux above $180 \mathrm{GeV}$ of $(3.73 \pm 0.41) \cdot 10^{-11} \mathrm{ph} \mathrm{cm}^{-2} \mathrm{~s}^{-1}$. The energy spectrum between $180 \mathrm{GeV}$ and $2 \mathrm{TeV}$ is compatible with a power law with a photon index $\alpha=2.72 \pm 0.14$ and is consistent with the quiescent state measured by HEGRA [41]. This is the first time 1ES 1959+650 has been observed down to $180 \mathrm{GeV}$. For the details of the analysis and results see [42].

\section{1ES 1218+304}

The AGN 1ES 1218+304 was observed several times with the Whipple telescope between 1995 and 2000. The obser- 


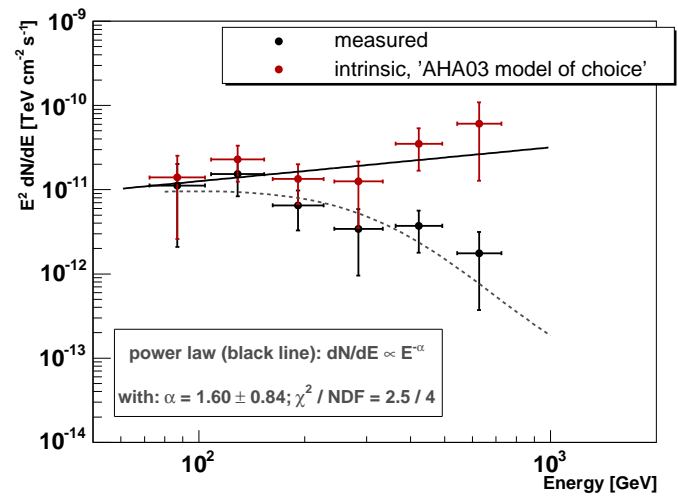

Fig. 7 Impact of the EBL on the differential energy spectrum of 1ES 1218+304. Black points: measured spectrum. Red points: reconstructed intrinsic spectrum of 1ES 1218+304 using the EBL density "model of choice" from [43], which is clearly excluded in [45]. Black line: pure power law fit to the intrinsic spectrum, the fit parameters are listed in the inlay.

vations resulted in an upper flux limit above $350 \mathrm{GeV}$ of $8.3 \cdot 10^{-12} \mathrm{ph} \mathrm{cm}^{-2} \mathrm{~s}^{-1}$ corresponding to $\sim 8 \%$ of the Crab Nebula flux [37]. The source was also observed by HEGRA between 1996 and 2002 and an upper flux limit above 840 $\mathrm{GeV}$ of $2.67 \cdot 10^{-12} \mathrm{ph} \mathrm{cm}^{-2} \mathrm{~s}^{-1}$ (or $12 \%$ of the Crab Nebula flux) was reported [34].

MAGIC observed 1ES $1218+304$ in seven nights in January 2005 (Table 1). The observed excess of 560 events has a statistical significance of 6.4 standard deviations above $140 \mathrm{GeV}$. 1ES $1218+304$ is the first source discovered by MAGIC. The nightly averaged $\gamma$-ray lightcurve did not show signs of significant variability. The energy spectrum was fitted with a pure power law with a photon index $\alpha=3.0 \pm 0.4$, and the determined flux level is below the upper limits at higher energies determined in the past. For details of the analysis and results see [44].

Measured VHE $\gamma$-ray spectra of distant sources can be used to derive constraints on the EBL density. Recently, using the VHE $\gamma$-ray spectra of two new detected HBL's at a similar redshift as 1ES $1218+304$, H.E.S.S. derived an upper limit on the EBL density between 1 and $4 \mu \mathrm{m}$ [45]. The main assumption needed to derive the limit is that the photon index of the intrinsic (de-absorbed) spectrum of an AGN can not be harder than 1.5. Though under debate [46], this assumption remains conservative if the VHE $\gamma$-rays are produced in shock regions. We did the same exercise using the 1ES 1218+304 spectrum. However, our result does not constrain the EBL density that strong compared to the upper limit that has been recently derived by H.E.S.S. due to large statistical errors of the measured spectral points and the fact that MAGIC did not measure flux points above $700 \mathrm{GeV}$ where the effect of the EBL becomes very significant. Fig. 7 illustrates the effect assumimg a relative high EBL density on the 1ES $1218+304$ spectrum. Though the de-absorbed spectrum is very hard, the fitted photon index $\alpha=1.6 \pm 0.8$ is within the allowed range $(>1.5)$.

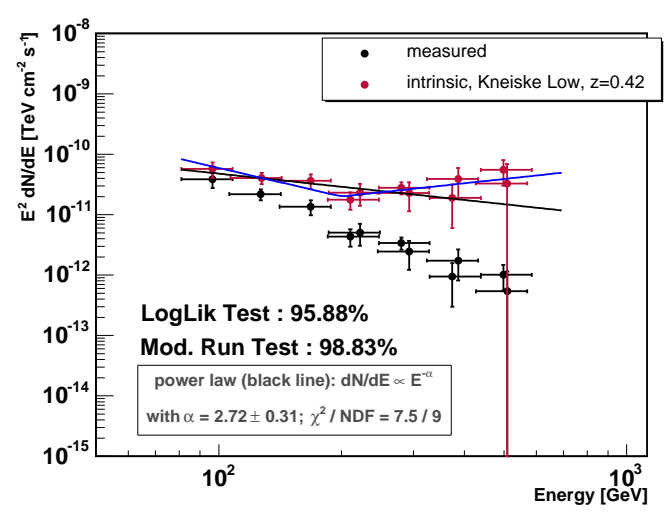

Fig. 8 Constraint on the redshift of PG 1553+113. Black points: measured combined differential energy spectrum of PG 1553+113 using MAGIC and H.E.S.S. data from 2005 and 2006. Red points: reconstructed intrinsic spectrum of PG 1553+113, using minimum possible density of the evolving EBL and the redshift of $\mathrm{z}=0.42$. Black line: power law fit to the intrinsic spectrum; the fit parameters are listed in the inlay. Blue line: broken power law fit.

\section{PG 1553+113}

PG $1553+113$ belongs to a catalog of X-ray bright objects [7] and, based on its SED properties, was one of the most promising candidates from a list of VHE $\gamma$-ray emitting AGNs proposed by [8]. So far, upper limits on the $\gamma$-ray emission have been reported by the Whipple collaboration (19\% Crab flux above $390 \mathrm{GeV}$ ) [47] and Milagro [48]. Recently the H.E.S.S. collaboration has presented evidence for a $\gamma$-ray signal at the $4 \sigma$ level (up to $5.3 \sigma$ using a low energy threshold analysis) above $200 \mathrm{GeV}$ corresponding to about $2 \%$ of the Crab flux [49].

PG 1553+113 was observed with the MAGIC telescope in 2005 at about the same time as the H.E.S.S. observations took place. Motivated by a hint of a signal in the preliminary analysis of the MAGIC data, additional observations were performed in 2006 (Table 11). Combining the data from 2005 and 2006, a very clear signal was detected with a total significance of $8.8 \sigma$. In $\gamma$-rays there was no evidence for short term variability on a time scale of days, but a significant change by a factor of three in the flux level from 2005 to 2006 was found. The combined 2005 and 2006 differential energy spectrum for PG $1553+113$ is well described by a pure power law with a photon index $\alpha=4.2 \pm 0.4$.

The signal detected by MAGIC confirms the tentative signal seen by H.E.S.S. at a higher energy threshold with data taken at about the same time as MAGIC in the 2005 period [49]. The source, therefore, can now be considered as a firmly established VHE $\gamma$-ray emitter.

An interesting aspect of the source is that attempts to determine its redshift from the optical observations failed so far. However, the VHE $\gamma$-ray spectrum of PG $1553+113$ can be used to derive an upper limit on the redshift of the source. Given the observed spectrum of PG 1553+113 and the minimum possible density of the evolving EBL (lower limit in [4]) we vary the distance of the source $(z)$ until the fit on 
the reconstructed intrinsic spectrum yields a photon index, which is beyond the allowed limit $(\alpha<1.5$, see also Section 8). Taking into account the statistical error on the fit and the systematic error on the slope (0.2) we derived an upper limit on the redshift of $z<0.78$. This value is compatible to the one reported in [49] $(z<0.74)$. For details of the analysis and results see [50].

An alternative method can be used to derive an upper limit on the redshift of PG 1553+113. The method is based on the indication that assuming rather large redshifts $(z>$ $0.3)$ the intrinsic spectrum of PG $1553+113$ seems to have a second component above $200 \mathrm{GeV}$. To prove this hypothesis (presence of a second component in the PG 1553+113 spectrum) we performed two different statistical tests on the reconstructed intrinsic spectrum. In the first test, we fitted the intrinsic spectrum with a pure power law (fit1) and with a broken power law (fit2). Then we used a likelihood ratio test [51] to determine the significance that fit 2 was a better hypothesis than fit 1 and not just by a coincidence. As the second test, a run test [51] was performed on the fit by a pure power law to the intrinsic spectrum. The run test was modified with the assumption of an equal number of data points below and above the fit. In order to increase the statistical power, we used the combined spectrum of MAGIC and H.E.S.S. data on PG $1553+113$ since the two spectra are in good agreement in the overlapping energy range. The reconstructed intrinsic spectrum was considered to have a significant second component at high energies in case both tests gave more than $2 \sigma$ confidence. An example is shown in Fig. 8 assuming a redshift of $z=0.42$. This is the smallest redshift where both tests gave more than $2 \sigma$ confidence for the presence of the second component. A detailed publication of this analysis is in preparation.

It cannot be excluded a priori that there is no second component in the spectrum of PG $1553+113$ above $200 \mathrm{GeV}$. However, in none of the measured VHE $\gamma$-ray spectra of extragalactic sources such component was found. Thus, we conclude that either this is the first time that an evidence of a second component in a VHE $\gamma$-ray spectrum was found or that the redshift of PG $1553+113$ is smaller than 0.42 .

\section{Conclusion and Outlook}

We gave an overview about outstanding findings based on MAGIC observations of extragalactic objects. The number of extragalactic VHE $\gamma$-ray sources detected by MAGIC is currently seven. Two of them, 1ES 1218+304 and Mkn 180 have been discovered by MAGIC. PG $1553+113$ has been co-discovered with H.E.S.S. In general, the reconstructed de-absorbed spectra seem to be the harder the further away the sources are, which might be related to an observational bias. Leptonic models (e.g. [6]) can describe the data, but there are exceptions like in case of 1ES 1959+650. For detailed modeling of the sources, extensive multiwavelength campaigns including radio through optical telescopes and $\mathrm{X}$-ray satellites are inevitable. In case of PG $1553+113$, we conclude that either the redshift of the source is $\mathrm{z}<0.42$ or for the first time a source with a clear evidence of a second emission component in the VHE $\gamma$-ray energy range has been observed. Re-observation of the presented sources as well as the analysis of further extragalactic objects is ongoing.

Acknowledgements We would like to thank the IAC for the excellent working conditions at the ORM in La Palma. The support of the German BMBF and MPG, the Italian INFN, the Spanish CICYT, ETH research grant TH 34/04 3, and the Polish MNiI grant 1P03D01028 is gratefully acknowledged.

\section{References}

1. Hartman, R. C. et al., ApJ Suppl. 123, 79-202 (1999)

2. Stecker, F. W. et al., ApJ 390, L49-L52 (1992)

3. Fazio, G.G. \& Stecker, F.W., Nature 226, 135 (1970)

4. Kneiske, T. M. et al., A\&A 413, 807-815 (2004)

5. Padovani, P. \& Giommi, P., ApJ 444, 567-581 (1995)

6. Maraschi, L., Ghisellini, G., \& Celotti, A., ApJ 397, L5-L9 (1992)

7. Donato, D. et al., A\&A 375, 739-751 (2001)

8. Costamante, L. \& Ghisellini, G., A\&A 384, 56-71 (2002)

9. Mannheim, K. et al., A\&A 315, 77-85 (1996)

10. Fossati, G. et al., NewAR 48, 419-422 (2004)

11. Holder, J. et al. ApJ 583, L9-L12 (2003)

12. Hartman, R. C. et al., ApJ 558, 583-589 (2001)

13. Sbarufatti, B., Treves, A., \& Falomo, R., et al., AJ 132, 1 (2006)

14. Rico, J., These Proceedings

15. Cortina, J. et al., In Proc. 29th Int. Cosmic Ray Conf. (Pune), 5, 359 (2005)

16. Daum, A. et al., APh 8, 1-11, (1997)

17. Wagner, R.M. et al., In Proc. 29th Int. Cosmic Ray Conf. (Pune), 4, 163 (2005)

18. Breiman, L., Machine Learning 45, 5-32 (2001)

19. Bock, R.K. et al., Nucl. Instr. Meth. A 516, 511-528 (2004)

20. Punch, M. et al., Nature 358, 477-478 (1992)

21. Gaidos, J. A. et al., Nature 383, 319-320 (1996)

22. Krennrich, F. et al., ApJ 575, L9-L13 (2002)

23. Aharonian, F. et al., A\&A 437, 95-99 (2005)

24. Krawczynski, H. et al., ApJ 559, 187-195 (2001)

25. Mazin, D. et al., In Proc. 29th Int. Cosmic Ray Conf. (Pune), 4, 331 (2005)

26. Primack, J. et al., AIP Conf. Proc. 745, 23 (2005)

27. Fazio, G.G. et al., ApJS 154, 39 (2004)

28. Elbaz, D. et al., A\&A 384, 848 (2002)

29. Albert, J. et al., Subm. to ApJ, astro-ph/0603478 (2006)

30. Quinn, J. et al., ApJ 456, L83 (1996)

31. Aharonian, F. et al., A\&A, 342, 69-86 (1999)

32. Perlman, E. S. et al., ApJ Suppl. 104, 251 (1996)

33. Catanese, M. et al., ApJ 501, 616 (1998)

34. Aharonian, F. et al., A\&A 421, 529-537 (2004)

35. Schroedter, M. et al., ApJ 634, 947-954 (2005)

36. Falco, E.F. et al., PASP 111, 438-452 (1998)

37. Horan, D. et al., ApJ 603, 51-61 (2004)

38. Dwek, E. \& Krennrich, F., ApJ 618, 657-674 (2005)

39. Albert, J. et al., ApJ in press, astro-ph/0606630 (2006).

40. Nishiyama, T. et al., In Proc. 26th Int. Cosmic Ray Conf. (Salt Lake City) 3, 370 (1999)

41. Aharonian, F. et al., A\&A 406, L9 (2003)

42. Albert, J. et al., ApJ 639, 761-765 (2006)

43. Aharonian, F., In Proc. 27th Int. Cosmic Ray Conf. (Hamburg), astro-ph/0112314 (2001)

44. Albert, J. et al., ApJ 642, L119-L122 (2006)

45. Aharonian, F. et al., Nature 440,1018 (2006)

46. Katarzyński, K. et al., A\&A 453, 47-56 (2006)

47. de la Calle Perez, I. et al., ApJ 599, 909 (2003)

48. Williams, D. In AIP Conf. Proc. 745, 499-504 (2005)

49. Aharonian, F. et al., A\&A 448, L19-L23 (2006)

50. Albert, J. et al., Subm. to ApJ, astro-ph/0606161 (2006)

51. Barlow, R. J.: Statistics. John W1ley \& Sons (1994) 